\title{
Some Results on the Analysis of Water Waves
}

\author{
Snehanshu Saha
}

\begin{abstract}
The analysis of water waves in general is an interesting study point in chemical and mechanical engineering. Ocean waves study is a branch of water wave models that has gained significant momentum in the last couple of decades. The paper investigates a phenomenon called "wave-breaking" which is one of the most intriguing longstanding problems of water wave theory. A certain class of equations namely Camassa-Holm and Degasperis-Procesi is generalized and new results on existence are obtained.
\end{abstract}

Index Terms-Local existence, blow-up, b-family of equations.

\section{INTRODUCTION}

For models describing water waves, wave breaking holds if the wave profile remains bounded, but it's slope becomes unbounded in finite time [11]. Breaking waves are commonly observed in the ocean and important for a variety of reasons, but surprisingly little is known about them. Indeed, breaking waves place large hydrodynamic loads on man-made structures, transfer horizontal momentum to surface currents, provide a source of turbulent energy to mix the upper layers of the ocean, move sediment in shallow water, and enhance the air-sea exchange of gases and particulate matter [3], [10]. To further understand why waves break and what happens during and after breaking themselves, we must first investigate the dynamics of wave breaking. Research work on breaking waves can be divided into three categories: those concerning waves before, during and after breaking. Although we are now understanding much about the processes leading up to breaking.The pertinent question is: What happens after breaking of those waves?

Consider the following partial differential equations.

$$
m_{t}+c_{0} m_{x}+\gamma u_{\{x x x\}}+u m_{x}+b m u_{x}=0,
$$

with $m=u-\alpha^{2} u_{x x}$

The Degasperis-Procesi (DP) [6,5] equation is a special case of the above

$$
y_{t}+y_{x} u+3 y u_{x}=0, \quad x \in \mathbb{R}, \quad t>0,
$$

with $y=u-u_{\{x x\}}$

The solution to the DP equation describes shock waves [7]. Liu and Lin [12], [13], [14] proved that the first blow-

Manuscript received April 17, 2012; revised May 28, 2012.

Dr. Snehanshu Saha is with PES School of Engineering (Phone: +918066186622, e-mail: snehanshusaha@pes.edu). up to the DP equation must occur as wave breaking and shockwaves are likely to appear afterwards. The other two integrable equations in the family are the Korteweg-de Vries $(\mathrm{KdV})(b=1)$ and and the Camassa-Holm $(\mathrm{CH})(b=2)$ shallow water equation [2], [4].These three cases exhaust in the completely integrable candidates for (1) by Painleve's analysis. For the $\mathrm{CH}$ equation, a procedure to understand the continuation of solutions beyond wave breaking has been constructed by Bressan and Constantin [1]. The paper investigates 'wave breaking' [11] by proving a result related to the blow up [7], [10] of solutions to a certain periodic profile.

\section{LOCAL WELL-POSEDNESS}

In this section, we apply Kato's theory to establish local well-posedness for the Cauchy problem. For convenience, we state here Kato's theorem in a form suitable for our purpose. Consider the abstract quasi-linear evolution equation

$$
\left\{\begin{array}{l}
\frac{d v}{d t}+A(v) v=f(v), \quad t \geq 0 \\
v(0)=v_{0}
\end{array}\right.
$$

Let $X$ and $Y$ be Hilbert spaces such that $Y$ is continuously and densely embedded in $X$, and let $Q: Y \rightarrow$ $X$ be a topological isomorphism. Let $L(Y, X)$ denote the space of all bounded linear operators from $\mathrm{Y}$ to $\mathrm{X}$. If $X=Y$, we denote this space by $L(X)$. The linear operator $A$ belongs to $G(X, 1, \beta)$ where $\beta$ is a real number, that is, -A generates a $C_{0}-$ semigroup such that $\left\|e^{-s A}\right\|_{L(X)} \leq e^{\beta s}$. Under the assumptions made by [8], [9], we state the following lemma:

\section{Lemma 2.1.}

(Kato, [8]) Assume that (i), (ii), and (iii) hold. Given $v_{0} \in Y$, there is a maximal $\mathrm{T}>0$ depending only on $\left\|\mathrm{v}_{0}\right\|_{Y}$ and a unique solution $\mathrm{v}$ to (1) such that $v=v\left(? v_{0}\right) \in C([0, T), Y) \cap C^{1}([0, T), X)$.Moreover, the map $v_{0} \mapsto v\left(? v_{0}\right)$ is a continuous map from $\mathrm{Y}$ to $C([0, T), Y) \cap C^{1}([0, T), X)$.

We begin by fixing some notations. All spaces of functions are assumed to be over $\mathbb{S}$, where $\mathbb{S}=\mathbb{R} \backslash \mathbb{Z}$ for simplicity, we drop $\mathbb{S}$, in our notation for function spaces if there is no ambiguity. If $A$ is an unbounded operator, we denote by $\mathrm{D}(\mathrm{A})$ the domain of $\mathrm{A}$. [A, B] 
denotes the commutator of two linear operators A and B.

With $\mathrm{m}=\mathrm{u}-\mathrm{u}_{x x}$, we consider the Cauchy problem

$$
\begin{gathered}
m_{t}+u m_{x}+b u_{x} m=0, \quad t>0, x \in \mathbb{S} \\
m(0, x)=u_{0}(x)-u_{0, x x}(x), \quad x \in \mathbb{S}, \\
\mathrm{u}(\mathrm{t}, \mathrm{x})=\mathrm{u}(\mathrm{t}, \mathrm{x}+1)
\end{gathered}
$$

Note that if $g(x):=\frac{1}{2} e^{-|x|}, x \in \mathbb{S} \quad, \quad$ then $\left(1-\partial_{x}^{2}\right)^{-1} f=g * f$ for all $f \in L^{2}(\mathbb{S}) \quad$ for $\quad$ all $f \in L^{2}(\mathbb{S})$ and $g * m=u$ where $*$ denotes convolution. Using this identity, we can rewrite (2) as a quasi-linear evolution equation of hyperbolic type:

$$
\begin{gathered}
u_{t}+u u_{x}+\partial_{x} g *\left(\frac{b}{2} u^{2}+\frac{3-b}{2} u_{x}^{2}\right)=0, \quad t>0, x \in \mathbb{S} \\
u(0, x)=u_{0}(x), \quad x \in \mathbb{S} \\
\mathrm{u}(\mathrm{t}, \mathrm{x})=\mathrm{u}(\mathrm{t}, \mathrm{x}+1)
\end{gathered}
$$

\section{Theorem 2.2}

For any constant $\mathrm{b}$, given $H^{s}(\mathbb{S}), s>\frac{3}{2}$, there exist a maximal $\mathrm{T}=\mathrm{T}\left(\mathrm{u}_{-}\{0\}\right)>0$ and a unique strong solution $\mathrm{u}$ to (3), such that:

$$
u=u\left(? u_{0}\right) \in C\left([0, T), H^{s}(\mathbb{S})\right) \cap C^{1}\left([0, T), H^{s-1}(\mathbb{S})\right)
$$

Moreover, the solution depends continuously on the initial data, i.e. the mapping

$$
u_{0} \mapsto u\left(? u_{0}\right): H^{s}(\mathbb{S}) \rightarrow C\left([0, T), H^{s}(\mathbb{S})\right) \cap C^{1}\left([0, T), H^{s-1}(\mathbb{S})\right)
$$

\section{Is continuous}

To prove this theorem, we apply Lemma 2.1 with

$$
A(u)=u \partial_{x}, f(u)=-\partial_{x} g *\left(\frac{b}{2} u^{2}+\frac{3-b}{2} u_{x}^{2}\right), Y=H^{s}, X=H^{s-1}, \Lambda=\left(1-\partial_{x}^{2}\right)^{\frac{1}{2}},
$$

$$
\text { And } Q=\Lambda^{1}[8]
$$

\section{Theorem 2.3}

The maximal $T$ in Theorem 2.2 may be chosen independent of $\mathrm{s}$ in the following sense. If

$u=u\left(? u_{0}\right) \in C\left([0, T), H^{s}\right) \cap C^{1}\left([0, T), H^{s-1}\right)$ to

and $\quad u_{0} \in H^{s^{\prime}}$ for some $s^{\prime} \neq s, s^{\prime}>\frac{3}{2}$, then $u \in C\left([0, T), H^{s^{\prime}}\right) \cap C^{1}\left([0, T), H^{s^{\prime}-1}\right)$ and with the same T. In particular, if $u_{0} \in H^{\infty}=\bigcap_{s \geq 0} H^{s}$, then $u \in C\left([0, T), H^{\infty}\right)$

\section{BLOW UP}

By using the local well-posedness result of Theorem (3) and energy estimates, the following precise blow-up scenario of strong solutions to (3) can be obtained.

\section{Theorem 3.1}

Assume $b \in \mathbb{R}$ and $u_{0} \in H^{s}(\mathbb{S}), s>\frac{3}{2}$. Then blow up of the strong solution $u=u\left(? u_{0}\right)$ in finite time $T<+\infty$ occurs if and only if

$$
\liminf _{t \uparrow T}\left\{(2 b-1) \inf _{x \in \mathbb{R}}\left[u_{x}(t, x)\right]\right\}=-\infty .
$$

Case 1: If $b=1 / 2$

\section{Proof:}

$$
\frac{d}{d t} \int_{\mathbb{S}} m^{2} d x=0 \Longrightarrow \frac{d}{d t} \int_{\mathbb{S}} m^{2} d x=\frac{d}{d t} \int_{\mathbb{S}} m_{0}^{2} d x
$$

On the other hand,

$$
\left|u_{x}\right| \leq \frac{1}{\sqrt{2}}\|u\|_{H^{2}} \leq \frac{1}{\sqrt{2}}\|m\|_{L^{2}}=\frac{1}{\sqrt{2}}\left\|m_{0}\right\|_{L^{2}}
$$

Then, By 3.2

\section{$\frac{d}{d t} \int_{\mathbb{S}} m_{x}^{2} d x=-2 \int_{\mathbb{S}} m_{x}^{2} u_{x} d x+\frac{1}{2} \int_{\mathbb{S}} m^{2} u_{x} d x \leq \sqrt{2}\left\|m_{0}\right\|_{L^{2}} \int_{\mathbb{S}} m_{x}^{2} d x+\frac{1}{2 \sqrt{2}}\left\|m_{0}\right\|_{L^{2}}^{3}$}

By means of Gronwall's inequality,

$\left\|m_{x}\right\|_{L^{2}}^{2} \leq\left(\left\|\partial_{x} m_{0}\right\|_{L^{2}}^{2}+\frac{1}{4}\left\|m_{0}\right\|_{L^{2}}^{2}\right) e^{s q r t 2\left\|m_{0}\right\|_{L^{2}} t}$

Case 2: if $b \neq \frac{1}{2}$ and if $u_{-} \mathrm{x}$ is bounded

Proof: Applying a simple density argument, we only need to show that the above theorem with some $s \geq 3$. Here we assume $\mathrm{s}=3$ to prove the above theorem. Multiplying (2) with $\mathrm{m}$ and integrating on $\mathbb{S}$ with respect to $\mathrm{x}$, we obtain:

$$
\frac{d}{d t} \int_{\mathbb{S}} m^{2} d x=-(2 b-1) \int_{\mathbb{S}} m^{2} u_{x} d x
$$

On the other hand, differentiating (2) with respect to $x$ and multiplying with $\mathrm{m}_{x}$, integrating on $\mathbb{S}$ with respect to $x$, and integrating by parts yield 
$\frac{d}{d t} \int_{\mathbb{S}} m_{x}^{2} d x=-(2 b+1) \int_{\mathbb{S}} m_{x}^{2} u_{x} d x+b \int_{\mathbb{S}} m^{2} u_{x} d x$.

It is thereby inferred from (3.2) and (3.3) that

$$
\frac{d}{d t} \int_{\mathbb{S}}\left(m^{2}+m_{x}^{2}\right) d x=-(2 b+1) \int_{\mathbb{S}} m_{x}^{2} u_{x} d x-(b-1) \int_{\mathbb{S}} m^{2} u_{x} d x .
$$

If $u_{x}$ is bounded from below on $[0, T) \times \mathbb{S}$, i.e., there exists $\mathrm{M}>0$ such that $-u_{x}(t, x) \leq M$ on $[0, T) \times \mathbb{S}$, then the relation (6) implies:

$\frac{d}{d t} \int_{\mathbb{S}}\left(m^{2}+m_{x}^{2}\right) d x \leq(2 b+1) M \int_{\mathbb{S}}\left(m^{2}+m_{x}^{2}\right) d x$.

And by means of Gronwall's inequality, we deduce that

$$
\int_{\mathbb{S}} m^{2}+m_{x}^{2} d x \leq\left(\int_{\mathbb{S}} m_{0}^{2}+m_{0 x}^{2} d x\right) e^{(2 b+1) M t}, \forall t \in[0, T) .
$$

Noting that,

$$
\|u(t)\|_{3} \leq\left(\int_{\mathbb{S}} m^{2}+m_{x}^{2} d x\right)^{1 / 2}
$$

and in view of (8), it follows that if $\left\{u_{x}(t)\right\}$ is bounded from below on $[0, \mathrm{~T})$, then the $H^{3}(\mathbb{S})$-norm of the solution to Eq.(3) is said not to have broken in finite time. This completes the proof of Theorem (4).

\section{REMARKS:}

It can be further shown that if $\mathrm{m}_{0}=\mathrm{u}_{0}-\mathrm{u}_{0 x x}$ doesn't change sign, then $m(t), \forall t$ will not change sign as long as $\mathrm{m}(\mathrm{t})$ exists. It is observed that if $\mathrm{u}(\mathrm{t}, \mathrm{x})$ is a solution to (3) with $\mathrm{u}(0, \mathrm{x})$ with $\mathrm{u}(0, \mathrm{x})=\mathrm{u}_{0} \mathrm{x}$, then $-\mathrm{u}(\mathrm{t},-\mathrm{x})$ is also a solution to (2.3) with initial datum $-\mathrm{u}_{0}(-\mathrm{x})$. Hence, due to the uniqueness of the solutions, the solution to (3) is odd as long as the initial datum $\mathrm{u}_{0} \mathrm{X}$ is odd. The blow up theorem is concerned with this data.

\section{REFERENCES}

[1] A. Bressan and A. Constantin, "Global conservative solutions of the CH equation," Archive for Rational Mechanics and Analysis, vol. 183, 2007, pp. 215-239

[2] R. Camassa and D. Holm, "An integrable shallow water equation with peaked solitons," Phys. Rev. Letters, vol. 71, 1993, pp. 16611664.

[3] E. D. Cokelet, Breaking Waves, Nature, vol. 267, 1997, pp. 769-774.

[4] H. R. Dullin, G. A. Gottwald, and D. D. Holm, "An integrable shallow water equation with linear and nonlinear dispersion," Phys. Rev. Letters, vol. 87, 2001, pp. 4501-4504.

[5] A. Degasperis, D. D Holm, and A. N. W Hore, "A new integral equation with peakon solutions," Theoretical and Mathematical Physics, 2002, pp. 133

[6] A. Degasperis and M. Procesi, "Asymptotic integrability," in Symmetry and Perturbation Theory, Edited by A. Degasperis and G. Gaeta, World Scientific, 1999, pp. 23-37.

[7] H. Lundmark, "Formation and Dynamics of Shock Waves in the DP Equation," Journal of Nonlinear Science, vol. 17, 2007, pp. 169-198

[8] T. Kato, "Quasi-linear equations of evolution, with applications to partial differential equations," in: Spectral Theory and Differential Equations, Lecture Notes in Math., Springer Verlag, Berlin, vol. 448, 1975, pp. 25-70.

[9] T. Kato, "On the Korteweg-de Vries equation," Manuscripta Math., vol. 28, 1979, pp. 89-99.

[10] D. H. Peregrine, "Breaking waves on beaches," Ann. Rev. Fluid Mech., vol. 15, 1983, pp. 149-178

[11] G. B. Whitham, Linear and Nonlinear Waves, J. Wiley and Sons, New York, 1980.

[12] Y. Liu and Z. Yin, "On the blow-up phenomenon for the DP Equation," Communications in Mathematical Physics, vol. 267, 2006, pp. 801-820

[13] Z. Yin, "On the Cauchy problem for an integrable equation with peakon solutions," Illinois J. Math., vol. 47, 2003, pp. 649-666.

[14] Z. Yin, "Well-posedness, blow-up, and global existence for an integrable shallow water equation," Discrete and Continuous Dynamical Systems, vol. 11, 2004, pp. 393-411. 